\title{
Loss of CD8 and TCR binding to Class I MHC ligands following $T$ cell activation
}

\author{
Charlly Kao, Mark A. Daniels ${ }^{1}$ and Stephen C. Jameson \\ Department of Laboratory Medicine and Pathology, Center for Immunology, University of Minnesota, \\ MMC 334, 420 Delaware Street SE, Minneapolis, MN 55455, USA \\ ${ }^{1}$ Present address: Department of Transplantation Immunology and Nephrology, University Hospital Basel, \\ $\mathrm{CH} 4031$ Basel, Switzerland
}

Keywords: activation, CTL, tetramer

\begin{abstract}
The capacity of $\mathrm{T}$ cells to bind peptide/MHC ligands changes with $\mathrm{T}$ cell development and differentiation. Here we study changes in peptide/MHC multimer binding following T cell activation. Surprisingly, T cell activation caused a marked reduction in specific peptide/MHC Class I multimer binding, which was distinct from transient TCR down-regulation, and was especially dramatic for engagement with low-affinity peptide/MHC ligands. Direct CD8-Class I interactions were also profoundly and rapidly impaired following T cell stimulation, even though surface CD8 $\alpha$ and CD8 $\beta$ levels were unchanged after activation, suggesting that decreased CD8 co-receptor binding contributes to this effect. Finally, we show that enzymatic desialylation restores much of the multimer binding on activated $T$ cells, suggesting that altered glycosylation may inhibit TCR/CD8 binding to peptide/MHC ligands. These radical changes in activated T cells' ability to perceive peptide/MHC ligands may contribute to selective outgrowth of clones with high affinity for the stimulatory ligand.
\end{abstract}

\section{Introduction}

Activation is thought to enhance the reactivity and sensitivity of $\mathrm{T}$ lymphocytes for their cognate peptide/MHC ligands. Various reports have suggested that, compared with naive cells, antigen-experienced effector and memory T cells require lower doses of their specific antigen/MHC ligand for activation, respond more quickly to these stimuli and/or have greater flexibility in detecting lower affinity variants of the antigenic ligand (1-4). Indeed, it has been estimated that CTL lysis can be provoked by engagement of 1-3 peptide/MHC ligands $(5,6)$. In one study on CD8 T cells, effector cells were superior to naive $T$ cells in their capacity to aggregate dimeric peptide/MHC ligands, an effect which appeared to correlate with changes in lipid raft content or distribution (7), perhaps providing a mechanism to explain heightened sensitivity of these cells. In addition, TCR stimulation on CTL clones leads to enhanced CD8 binding to Class I MHC molecules directly, in the absence of TCR recognition of the peptide-MHC complex (also called 'non-cognate' CD8 binding) $(8,9)$. We and others demonstrated that immature thymocytes are more efficient than mature $T$ cells at binding non-cognate Class I $\mathrm{MHC}$ molecules, a property which appears to correlate with lower levels of cell-surface sialylation on immature $T$ cells $(10,11)$. Interestingly, the fact that activated T cells also have lowered levels of surface sialylation (12-16) might suggest that they too would be enhanced for non-cognate CD8 binding.

On the other hand, some groups have reported that stimulation of $T$ cells reduces their ability to bind specific peptide/MHC multimers. While in some cases, a reduction in multimer binding might simply be a consequence of the well-known phenomenon of TCR down-regulation following T cell activation $(3,17)$, this appears not to explain the loss of multimer binding in several studies (18-20). In one elegant series of studies, activated T cells specific for sub-dominant influenza epitopes were shown to be unable to bind specific peptide/MHC multimers, although TCR and CD8 expression levels were found to be normal $(21,22)$. Furthermore, reduced multimer binding could be induced by disruption of lipid raft domains even though TCR and CD8 levels were maintained (22), indicating that multimer binding can be compromised despite sustained surface expression of TCR and CD8.

Therefore, it is currently unclear whether $\mathrm{T}$ cell activation leads to enhanced or diminished peptide/ $\mathrm{MHC}$ ligand binding, with reports supporting both conclusions.

We previously used peptide/MHC Class I multimers to explore the requirement for CD8 in binding to cognate TCR
Correspondence to: S. C. Jameson; E-mail: james024@umn.edu

Transmitting editor: $\mathrm{H}$. Ploegh
Received 14 September 2005, accepted 22 September 2005 Advance Access publication 1 November 2005 
1608 Loss of peptide/MHC binding with T cell activation

ligands on naive T cells (23), and also to study the developmental regulation of CD8 non-cognate binding (10). These and other studies suggested that there is considerable variability in multimer staining depending on the maturation state of the $T$ cell and the participation of the CD8 $\alpha \beta$ coreceptor $(10,11,24,25)$. In this report, we studied the changes in peptide/MHC multimer binding which accompany $T$ cell activation. We make the surprising finding that the capacity of activated CD8 T cells to bind cognate multimers is severely depressed for several days following stimulation, even after TCR expression has returned to normal levels. We find that the loss of cognate binding is more dramatic and sustained when low-affinity TCR ligands are studied, suggesting that activated CD8 T cells may preferentially lose sensitivity to weak agonists. These changes in tetramer binding are in part due to alterations in CD8-Class I binding, since we observe a precipitous decline in non-cognate multimer binding with $\mathrm{T}$ cell stimulation, despite the fact that CD8 $\alpha$ and CD8 $\beta$ expression levels are maintained after $T$ cell activation. Thus, our data suggest that CD8 T cell activation induces a state of impaired engagement with Class I MHC-peptide complexes, which compromises recognition of all but the highest affinity ligands.

\section{Methods}

\section{Mice and cells}

C57BL/6 mice were obtained from Jackson Laboratory (Bar Harbor, ME, USA). OT-I and 2C mice were generated and maintained under specific pathogen-free conditions. Major lymph nodes and/or spleens were harvested, and single-cell suspensions were created by mashing over a metal mesh screen immersed in HBSS (GIBCO), filtered and then centrifuged and re-suspended in 'RP10' medium (RPMI 1640 from GIBCO containing 10\% FCS plus $5 \mathrm{mM}$ HEPES, $50 \mathrm{U}$ penicillin, $50 \mu \mathrm{g} \mathrm{ml}^{-1}$ streptomycin and $50 \mu \mathrm{g} \mathrm{ml}^{-1}$ gentamicin sulfate, $50 \mu \mathrm{M}$ 2-mercaptoethanol and $2 \mathrm{mM}$ glutamine). Cells were used for subsequent experiments and cultures without further purification.

\section{Peptides and MHC multimers}

The peptides ovalbumin peptide (OVA) (SIINFEKL), G4 (SIIGFEKL), SIY (SIYRYYGL), SIY-A6 (SIYRYAGL), VP10 (STLNFNNL) and P815p (HIYEFPQL) were synthesized by Research Genetics or Invitrogen. $\mathrm{K}^{\mathrm{b}}-\beta 2 \mathrm{~m}$-peptide complexes were made as described previously (23) from procedures adapted from Busch and Altman $(26,27)$. Biotinylated monomers were concentrated to $1 \mathrm{mg} \mathrm{ml}^{-1}$ and stored in $10-100 \mu \mathrm{l}$ aliquots at $-80^{\circ} \mathrm{C}$. Multimers were prepared by mixing with regular grade streptavidin-phycoerythrin (SA-PE) conjugate (Molecular Probes, Eugene, OR, USA) at an 8:1 molar ratio (1:1 mass ratio) and incubating at room temperature for 30-60 min prior to staining. The mass concentration of multimers was assumed to be $\sim 2 \times$ the mass concentration of SA-PE (e.g. $0.5 \mathrm{mg} \mathrm{ml}^{-1}$ of SA-PE yields $\sim 1 \mathrm{mg} \mathrm{ml}^{-1}$ multimers). Multimers were used at $2-50 \mu \mathrm{g} \mathrm{ml}^{-1}$ without further purification. We found that multimer staining intensity, especially with low-affinity peptide/MHC ligands, decreased with prolonged storage of pre-formed multimers. Hence for optimal sensitivity, non-cognate and low-affinity multimer binding assays were performed within $1-2 \mathrm{~h}$ after multimerization.

\section{Antibodies, multimer staining and flow cytometry}

T cells $\left(0.5 \times 10^{6}\right.$ to $\left.1 \times 10^{6}\right)$ were stained on ice with multimers in $100 \mu$ of FACS buffer (PBS, 1\% FCS and $0.2 \%$ sodium azide) for 30 min (FACS buffer conditions) or RP10 for 2 h (RP10 conditions). Standard staining conditions include multimers at $10 \mu \mathrm{g} \mathrm{ml}^{-1}$ (23), which gives staining close to saturating levels (data not shown), but higher or lower concentrations were used in some experiments, as indicated. Staining at $4^{\circ} \mathrm{C}$ was used to enhance the low-affinity interactions studied, since preliminary experiments suggested that staining at $37^{\circ} \mathrm{C}$ decreased low-affinity multimer binding, similar to the observations of Whelan et al. (28), and also raised background, non-CD8 binding (data not shown). In some experiments, CD8 dependence for multimer binding was tested by inclusion of the blocking anti-CD8 $\alpha$ antibody, CT-CD8 $\alpha$ (Caltag Laboratories), which was used at $5 \mu \mathrm{g} \mathrm{ml}^{-1}$ of FITC-conjugated antibody added immediately prior to the multimer. For all other multimer stains, we gated on CD8+ cells in flow cytometric analyses by using the anti-CD8 $\alpha$ antibody, 53-6.7 (5 $\mathrm{g} \mathrm{m} \mathrm{ml}^{-1}$. FITC conjugated) (PharMingen or Biolegend). The 53-6.7 is known to enhance Class I MHC multimer binding (23), but it does not influence prebound multimers (data not shown). Therefore, with the exception of Fig. 6 (as indicated), cells were stained first with multimers and then washed and stained with 53-6.7. All surface staining aside from the multimer stains were done for $30 \mathrm{~min}$ on ice in PBS + 10\% FCS. Anti-CD8 $\beta$ (53-5.8), anti-V $\alpha 2$ (B20.1) and anti-V $\beta 8$ (F23.1) antibodies were purchased from PharMingen or Biolegend and used at $1-2 \mu \mathrm{g} \mathrm{ml}^{-1}$. Peanut agglutinin (PNA)-FITC was purchased from Vector Laboratories and used at $0.5 \mu \mathrm{g} \mathrm{ml}^{-1}$. Cells were analyzed on a Becton Dickinson FACSCalibur ${ }^{T M}$ or LSRII, and data were analyzed using FlowJo (TreeStar, San Carlos, CA, USA) software.

\section{In vitro T cell stimulation}

Unpurified OT-I lymph node cells $\left(8 \times 10^{5}\right)$ and $2 \times 10^{5}$ C57BL/6 $\left(\mathrm{H}-2^{\mathrm{b}}\right)$ unpurified splenocytes in $200 \mu \mathrm{RP} 10$ containing the indicated doses of OVA were added to 96well flat-bottom tissue culture treated wells and spun down briefly (300-400 $\times$ g, 1 min). For Figs 3 and 4, OT-I or 2C lymph node T cells $\left(25 \times 10^{6}\right)$ and $5 \times 10^{6} \mathrm{C} 57 \mathrm{BL} / 6$ splenocytes were cultured in $10 \mathrm{ml}$ RP10 in petri dishes (100 mm diameter) with 10 nM OVA (for OT-I cells) or $1 \mu \mathrm{M}$ SIY peptide (for 2C cells). Cell cultures were maintained in $37^{\circ} \mathrm{C}$ incubators under $5 \%$ $\mathrm{CO}_{2}$ tension for the times indicated. Media were changed daily starting at 2-3 days post-stimulation and IL-2 (10 $\mathrm{ng} \mathrm{ml}^{-1}$; Biosource) was added at the same time. Cultures were split as necessary from day 2 of activation.

\section{TCR blockade of multimer staining}

Naive OT-I T cells $\left(0.5 \times 10^{6}\right.$ to $\left.1 \times 10^{6}\right)$ were incubated with the $\mathrm{V} \alpha 2$-specific blocking antibody (B20.1) at various doses (78 $\mathrm{ng} \mathrm{ml}^{-1}-5 \mu \mathrm{g} \mathrm{ml}^{-1}$ ) in $100 \mu \mathrm{l}$ of RP10 for 30 min at $4^{\circ} \mathrm{C}$. Cells were washed with RP10 and stained with $1 \mu \mathrm{g} \mathrm{ml}^{-1}$ PE-conjugated B20.1 (PharMingen or Biolegend) for $30 \mathrm{~min}$ at $4^{\circ} \mathrm{C}$ in RP10 to detect remaining levels of unbound TCR. 
In parallel, naive or activated OT-I cells were stained with OVA $/ \mathrm{K}^{\mathrm{b}}$ multimers $\left(2 \mu \mathrm{g} \mathrm{ml}^{-1}\right)$, and binding was measured by flow cytometry as described above.

\section{Neuraminidase treatment}

Cells were treated with neuraminidase as previously described (10). Briefly, cells were prepared as above, washed one to two times in HBSS and re-suspended at $1 \times 10^{6}$ to $2 \times 10^{6} \mathrm{ml}^{-1}$ in HBSS. Following pre-incubation at $37^{\circ} \mathrm{C}$ for 15 min, neuraminidase (0.0095 units per 1-2 million cells) from Vibrio cholerae (Sigma, St Louis, MO, USA) was added, and the treated cells were incubated at $37^{\circ} \mathrm{C}$ for $20-40 \mathrm{~min}$. Cells were washed one to two times with RP10 before staining with the indicated multimers. Controls were processed in the same way without addition of neuraminidase.

\section{Results}

Specific peptide/MHC binding to OT-I CD8+ T cells is impaired following activation, despite high levels of surface TCR

We wished to investigate how $\mathrm{T}$ cell activation influenced peptide/MHC binding. Class I MHC-restricted OT-I TCR transgenic $T$ cells were activated in vitro, and then assessed for their ability to bind specific Class I MHC multimers. Within 24-h post-activation, we observed a dramatic loss in OT-I binding to the multimeric Class I MHC ligand, OVA $/ K^{\mathrm{b}}$ (Fig. 1A). The loss in multimer binding at this time point might be explained by the well-documented phenomenon of TCR down-regulation that accompany T cell activation $(17,29,30)$ (Fig. 1A). However, we were surprised to observe that OVA $/ \mathrm{K}^{\mathrm{b}}$ binding was still impaired at days 3 and 4 after activation, despite the fact that surface TCR expression had returned to the levels seen on naive OT-I cells (Fig. 1A). In addition, we studied binding of multimers loaded with G4 peptide, a variant of OVA that has lower affinity for the OT-I TCR (31). Binding of G4/K multimers (even when offered at high dose) was even more severely impaired than binding of the higher affinity OVA $/ K^{b}$ ligand (Fig. 1A). Moreover, loss of $\mathrm{G} 4 / \mathrm{K}^{\mathrm{b}}$ binding was sustained through days $4-5$ of activation, while binding of OVA $/ K^{\mathrm{b}}$ was substantially restored at these time points (Fig. 1A and data not shown). These findings were observed over a range of multimer doses. As shown in Fig. 1(B), titration of both OVA/K ${ }^{b}$ and $\mathrm{G} 4 / \mathrm{K}^{\mathrm{b}}$ multimers showed a consistent result in that specific multimer binding to activated OT-I cells was severely reduced compared with unstimulated OT-I cells.

The above experiments suggested that $T$ cell activation caused a disproportionate loss in Class I antigen binding that could not be explained by TCR down-regulation since the percent loss in multimer binding exceeded the percent loss in TCR expression levels and multimer binding was still impaired following recovery of surface TCR levels (Fig. 1). However, since TCR affinities for specific peptide/MHC ligands are low, efficient multimer binding is expected to be highly sensitive to the density of surface TCR. Thus, it was possible that a slight decrease in TCR levels would cause a disproportionate loss in multimer binding.

We tested this idea by performing a TCR blockade experiment to mimic TCR down-regulation. Naive OT-I cells were coated with increasing doses of a blocking TCR antibody (B20.1) to progressively inhibit the capacity of surface OT-I TCRs from binding multimers. The amount of remaining available TCR was then quantitated by measuring the level of staining with the PE-labeled B20.1 antibody. Alternatively, the antibody-coated naive OT-I cells were stained with OVA $/ K^{b}$ multimers. This allowed us to determine a relationship between available surface TCR and multimer binding, as shown in Fig. 2(A). Blockade with the B20 antibody led to efficient blockade of the OT-I TCR and, at high doses, resulted in loss of $>99 \%$ of OVA $/ \mathrm{K}^{\mathrm{b}}$ multimer binding to naive OT-I cells (Fig. 2A). Next, we compared multimer staining on activated OT-I cells (which were not treated with anti-TCR antibody) in comparison with naive OT-I cells subjected to TCR blockade. We observed that, even at equivalent levels of accessible TCRs, the multimer binding on activated OT-I cells was lower than that on naive Tcells (Fig. 2B). For example, activation with $1 \mathrm{nM}$ OVA resulted in loss of $\sim 50 \%$ of surface TCR by day 1 , yet cognate multimer staining was reduced by $~ 98 \%$ (Figs 2B and $\mathrm{C}$ ). We note some variation between experiments in the magnitude of the TCR down-regulation and loss of multimer binding, especially in cultures activated with low doses of antigen (data not shown), but the pattern shown is highly consistent. Thus, we reliably observed that OVA $/ \mathrm{K}^{\mathrm{b}}$ binding was $~ 5$ - to 10-fold lower on activated OT-I cells compared with antibody-blocked naive cells with similar levels of available OT-I TCR (Fig. 2C). These data again suggest that activation impairs ligand binding beyond levels expected from TCR down-regulation, and that changes in TCR levels alone do not account for the loss of multimer binding.

\section{Role of CD8 in the activation-induced loss in peptide/MHC binding}

We next tried to determine the underlying mechanism for this activation-induced phenomenon. Previous work has demonstrated a critical role for CD8 in binding cognate multimers on naive OT-I cells $(23,32)$. Subsequent work has shown that CD8 binding to non-antigen Class I (i.e. noncognate binding) can be detected using peptide/MHC multimers. This binding is considerably weaker on mature, naive CD8+ $T$ cells compared with immature CD4+8+ thymocytes $(10,11,24,25)$. However, we recently established methods which enhance detection of non-cognate binding on naive $T$ cells (see experimental procedures). Using these improved staining conditions, non-cognate multimer staining of naive OT-I cells could be detected.

We activated naive OT-I cells as described above and stained the cells with non-cognate $\left(\mathrm{P} 815 / \mathrm{K}^{\mathrm{b}}\right)$ multimers on successive days. The binding of CD8 to Class I multimers dropped to nearly undetectable levels within the first day after activation, and this phenomenon persisted throughout the time course (Fig. 3A). Importantly, this loss in non-cognate multimer binding could not be attributed to changes in surface levels of either CD8 $\alpha$ or CD8 $\beta$. Consistent with other reports (3), CD8 expression remained relatively constant throughout the activation (Fig. 3 and data not shown). This result implies that the drop in Class I MHC multimer binding is a result of changes in the 'quality' of the CD8 molecules, not the quantity of its expression. 
A
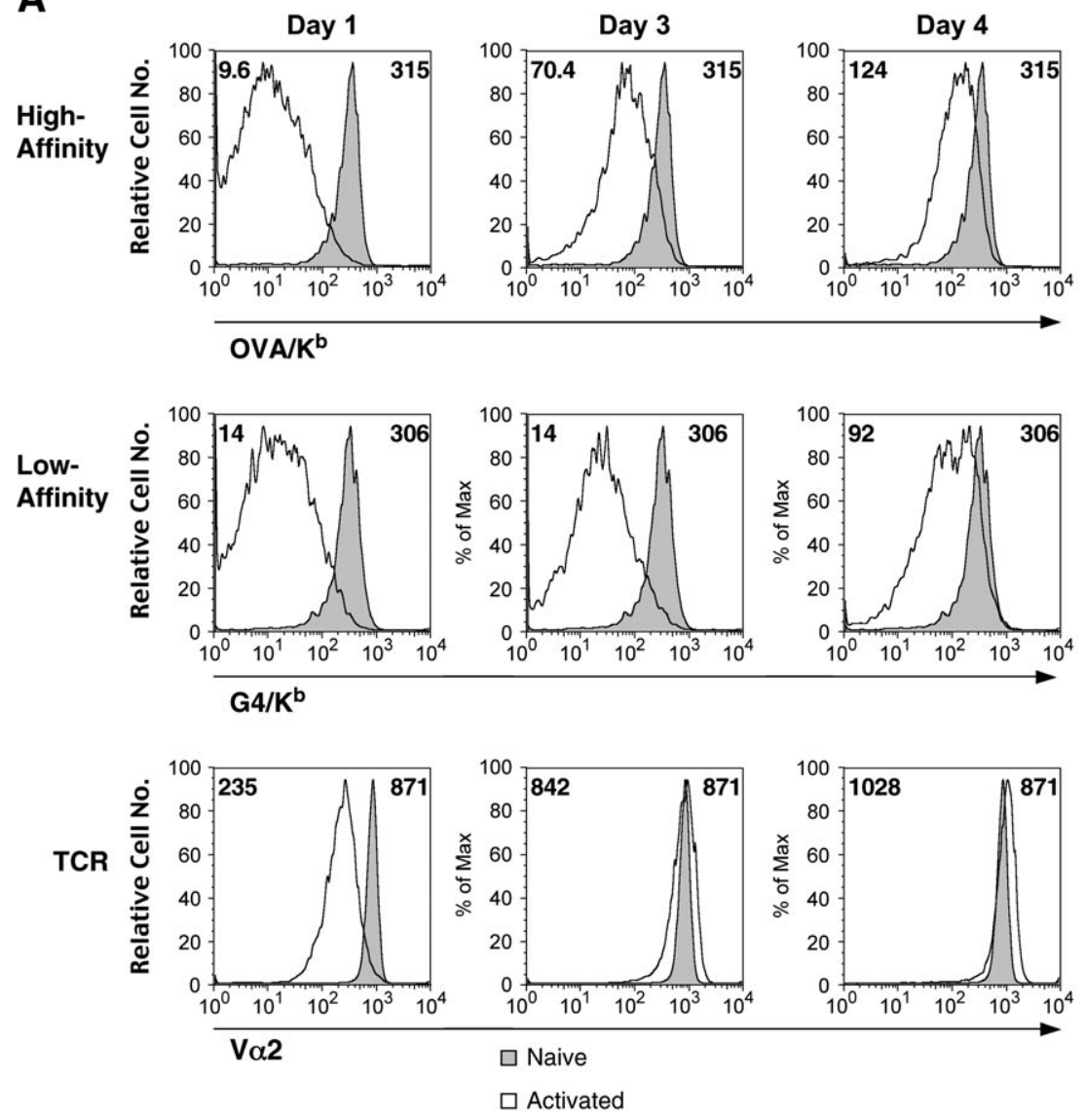

B

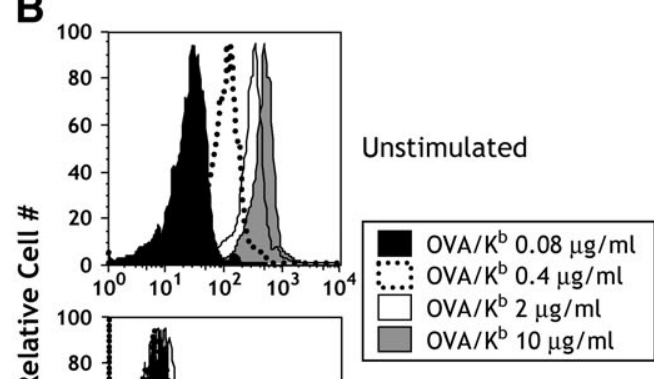

Activated

Unstimulated

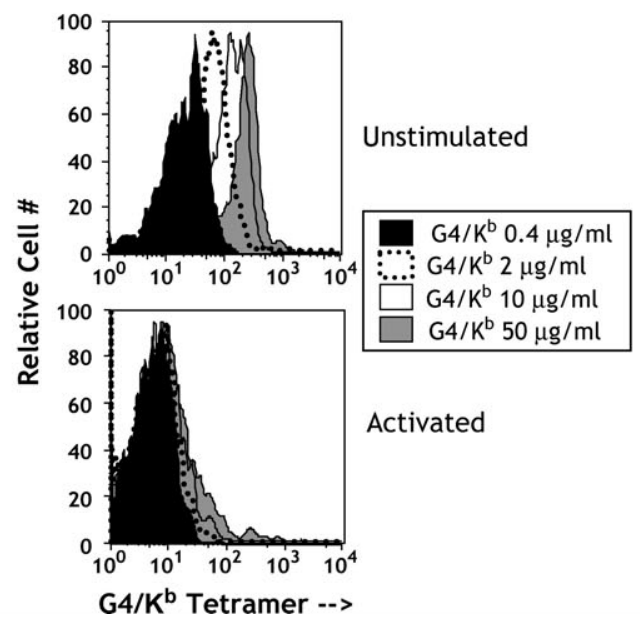

Fig. 1. Binding to specific peptide/MHC ligands is impaired on activated CD8 T cells, despite normal TCR levels. OT-I cells were stimulated in vitro with $1 \mu \mathrm{M}$ OVA. In (A) cells were assessed for binding to high-affinity $\left(\mathrm{OVA} / \mathrm{K}^{\mathrm{b}}\right)$ or low-affinity $\left(\mathrm{G} 4 / \mathrm{K}^{\mathrm{b}}\right)$ Class I MHC multimers over several days of activation, compared with naive OT-I. Staining was performed using $2 \mu \mathrm{g} \mathrm{ml} \mathrm{l}^{-1}$ of multimer in FACS buffer (for OVA $/ \mathrm{K}^{\mathrm{b}}$ ) or $10 \mu \mathrm{g} \mathrm{ml} \mathrm{I}^{-1}$ of multimer in RP10 media $\left(\mathrm{G} 4 / \mathrm{K}^{\mathrm{b}}\right)$, to attain similar levels of overall multimer staining. OT-I TCR levels were measured with the B20.1 specific for the Va2 chain. Representative histograms from days 1, 3 and 4 post-activation are shown. Median fluorescence intensities (MFIs) for activated cells are listed in the upper left corner and naive MFIs on the upper right corner. In (B) unstimulated or day 1 activated OT-I were stained with titered doses of OVA/K ${ }^{b}$ multimers (in FACS buffer) or G4/K $/ \mathrm{K}^{\mathrm{b}}$ multimers (in RP10) at the doses indicated. Activation-induced loss of OT-I TCR expression was similar to that shown in (A) (data not shown).

We were also interested in determining how early this phenomenon can be detected after activation. We observed changes in non-cognate multimers at 8-h post-activation (Fig. 3B). These data argue against a model in which the loss in multimer avidity was due to changes in cell size since cells at this time point have not begun blasting (data not shown). We detected a slight drop in non-cognate multimer binding when OT-I cells were cultured without antigen stimulus at this time point, which may be attributable to a slight loss of CD8 $\beta$ levels (Fig. 3B); however, the loss in binding due to activation is much more pronounced. It is important to note that loss of cognate binding is also severely impaired at $8 \mathrm{~h}$ of activation, however TCR down-regulation is also quite marked at this time point (data not shown).

Hence, these data suggested that T cell activation leads to a profound, rapid and sustained loss in CD8-Class I binding.

\section{Activation induces loss of TCR-and CD8-Class I binding by $2 \mathrm{C} \mathrm{CD8}+\mathrm{T}$ cells}

While the data above implicated changes in CD8 binding as a basis for the loss of specific peptide/MHC binding by activated OT-I cells, we wanted to determine whether CD8independent binding to the TCR was also influenced by activation.

For this, we turned to the 2C TCR transgenic system. As we and others have shown previously, 2C TCR binding to the SIY/K $K^{b}$ ligand can be detected even when CD8 molecules are absent or blocked $(23,32)$. Hence, to look at multimer binding in the absence of CD8 co-receptor participation, we stained resting and activated $2 \mathrm{C}$ cells with $\mathrm{SIY} / \mathrm{K}^{\mathrm{b}}$ multimers in the presence of the blocking CT-CD $8 \alpha$ antibody. This antibody inhibits CD8-dependent multimer staining $(23,32)$. Activated $2 \mathrm{C}$ CD8 T cells showed a decline in TCR binding to 
A

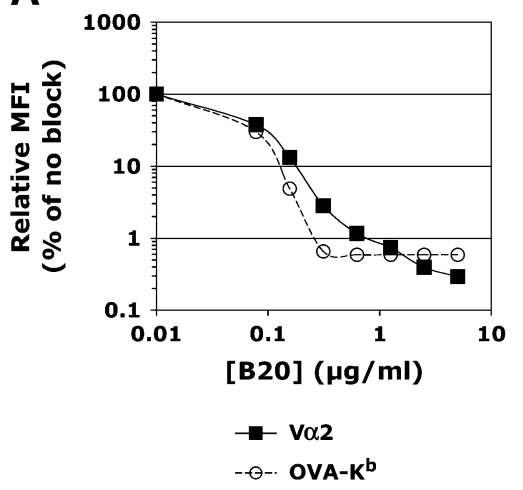

B


C


Fig. 2. Mimicking TCR down-regulation by antibody blockade on naive OT-I cells reveals an additional activation-induced effect that impacts peptide/MHC multimer binding. (A) Naive OT-I T cells were coated with increasing doses of the blocking monoclonal anti-V $\alpha 2$ antibody, B20.1, and then stained with OVA $/ \mathrm{K}^{\mathrm{b}}$ multimer $\left(2 \mu \mathrm{g} \mathrm{ml}{ }^{-1}\right.$ in FACS buffer) to assess antigen binding or with a PE-labeled B20.1 to measure remaining unbound levels of surface TCR. (B and C) OT-I cells were activated with $1 \mu \mathrm{M}$ and $1 \mathrm{nM}$ OVA, respectively. Relative median fluorescence intensities (MFIs) of bound OVA $/ \mathrm{K}^{\mathrm{b}}$ multimers or TCR levels were plotted on line graphs in the top panels. The bar graphs in the bottom panels compare OVA $/ \mathrm{K}^{\mathrm{b}}$ staining between cells that show an equivalent loss in TCR levels due to activation (day 1) or surface blockade with B20.1 described in (A).

SIY $/ K^{b}$ multimers in these assays (Fig. 4). The kinetics of multimer loss correlated with the drop in TCR levels at days 1 and 2 of post-activation, but the magnitude of the loss in surface TCR did not match that of the reduction in multimer staining (Fig. 4). Furthermore, TCR levels were similar to those on naive $2 \mathrm{C}$ cells at day 3 of activation, yet CD8-independent multimer binding is still only $60 \%$ of naive, suggesting that activation influenced the ability of the TCR to interact with its ligand. We saw similar results using SIY/K ${ }^{\mathrm{b}} \mathrm{D} 227 \mathrm{~K}$ multimers, which bear a crippling mutation in the CD8-binding site (data not shown). While we had hoped to analyze the CD8-negative $2 \mathrm{C}$ population in these experiments, these cells were poorly stimulated and rapidly lost in our activation cultures, limiting our ability to track their multimer-binding potential (data not shown).

We also studied $2 \mathrm{C}$ binding to SIY-A6/K $\mathrm{K}^{\mathrm{b}}$ multimers. SIY-A6/K was defined as an antagonist for $2 \mathrm{C} \mathrm{T}$ cells (33), and is presumed to bind the $2 \mathrm{C}$ TCR with a lower affinity than SIY. Unlike $\mathrm{SIY} / \mathrm{K}^{\mathrm{b}}, 2 \mathrm{C}$ staining with SIY-A6/ $\mathrm{K}^{\mathrm{b}}$ multimers is highly dependent on CD8 (Fig. 4) (34). Similar to the OT-I system, cognate multimer binding to this low-affinity ligand was drastically impaired following $2 \mathrm{C}$ activation, and this effect was maintained over several days, even after TCR expression had returned to the same levels as naive $2 \mathrm{C}$ cells (Fig. 4). As with OT-I cells, CD8 $\alpha \beta$ levels were not notably changed on activated 2C cells, yet noncognate binding by $2 \mathrm{C} \mathrm{T}$ cells (to OVA $/ \mathrm{K}^{\mathrm{b}}$ multimers) was drastically reduced (data not shown).

Hence, these data suggest that both CD8-Class I and TCRClass I interactions with specific peptide/MHC ligands are compromised following $T$ cell activation, but argue that CD8dependent multimer binding is much more severely impacted.

\section{Activation-induced loss in multimer binding is partially attributable to surface sialylation}

Our laboratory and that of others have previously demonstrated that cell-surface sialylation is inversely correlated with non-cognate Class I multimer binding $(10,11)$. We examined the effects of surface sialylation on non-cognate multimer binding after activation using $V$. cholera neuraminidase, a broad range sialidase which cleaves sialic acid from many types of glycan adducts, including CD8 itself $(13,35)$. Hence, we tested whether neuraminidase would also influence multimer binding to activated CD8 T cells.

OT-I lymph node cells were activated with OVA in vitro for $60 \mathrm{~h}$ and stained with cognate and non-cognate multimers. As expected, OT-I activation led to notable loss of OVA $/ \mathrm{K}^{\mathrm{b}}$ multimer binding and profound decrease in binding of G4/K $/ \mathrm{K}^{\mathrm{b}}$ (low-TCR affinity ligand) and $\mathrm{P} 815 / \mathrm{K}^{\mathrm{b}}$ (non-cognate ligand) (Fig. 5). 



$\mathrm{P} 815 / K^{\mathrm{b}}$ (non-cognate)
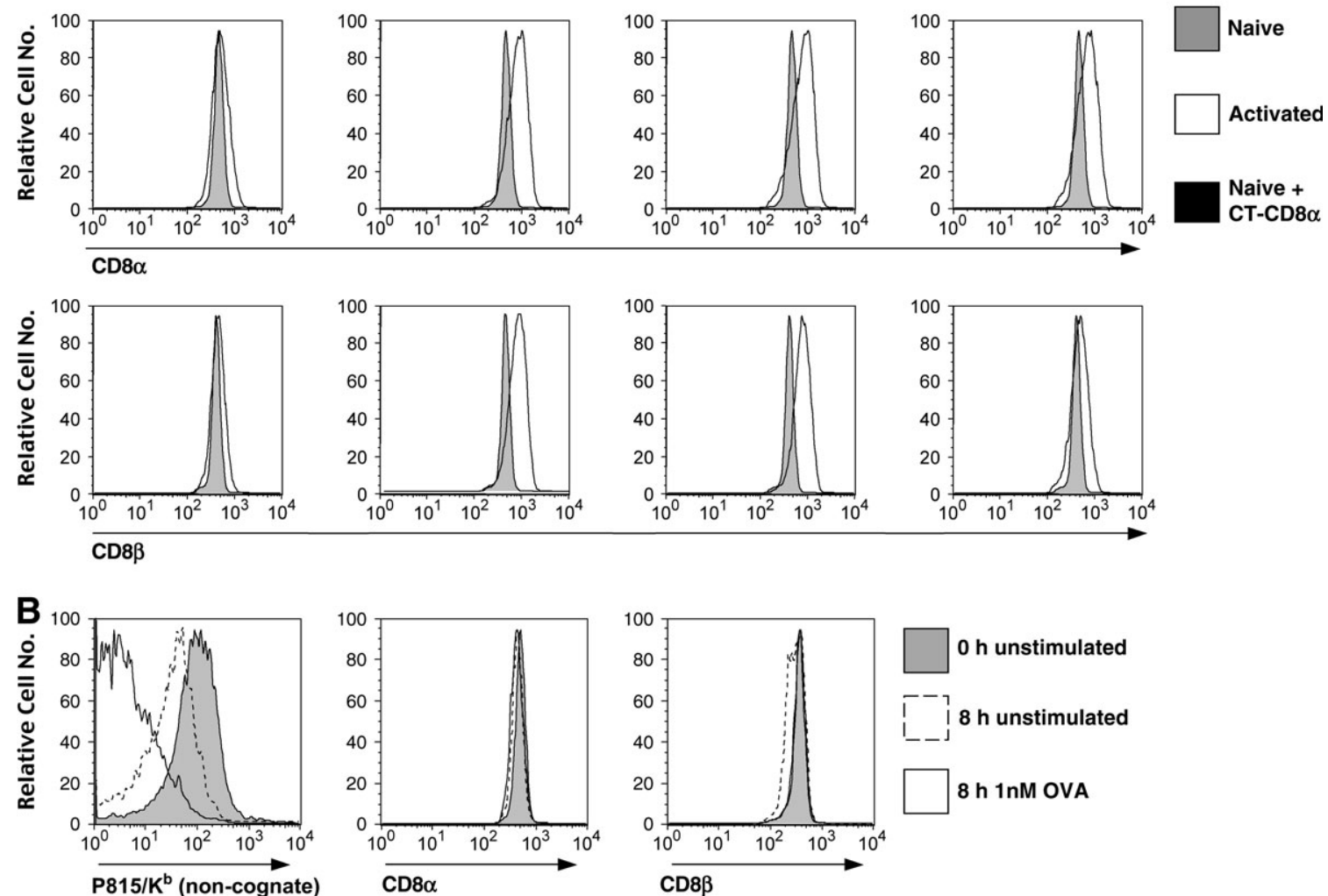

Fig. 3. Down-regulation of CD8 avidity for non-cognate Class I MHC on activated OT-I is rapid and sustained. (A) OT-I cells were activated in vitro with $10 \mathrm{nM}$ OVA, and non-cognate P815/K ${ }^{\mathrm{b}}$ multimer binding and CD8 levels were measured daily from days 1 to 5 post-activation. The monoclonal anti-CD $8 \alpha$ antibody, CT-CD8 $\alpha$, was used to block non-cognate binding on naive cells and shown as a baseline for comparison in the top row. (B) OT-I cells were activated as described above and assessed for non-cognate binding and CD8 levels 8-h post-stimulus. The staining for cells cultured ex vivo for $8 \mathrm{~h}$ without antigen stimulation is also shown. In all cases, staining used $50 \mu \mathrm{g} \mathrm{ml} \mathrm{l}^{-1} \mathrm{P} 815 / \mathrm{K}^{\mathrm{b}}$ multimer in RP10 at $4^{\circ} \mathrm{C}$.

In parallel, we treated both naive and the activated OT-I cells with neuraminidase, as described previously (10). Intriguingly, both cognate and non-cognate multimer binding were substantially enhanced by neuraminidase treatment of the activated OT-I cells (Fig. 5). Binding of OVA $/ \mathrm{K}^{\mathrm{b}}$ and, to an even greater extent, $\mathrm{G} 4 / \mathrm{K}^{\mathrm{b}}$ multimers was enhanced by desialylation of activated OT-I cells, and binding approached the levels seen on desialylated naive OT-I cells. Even after neuraminidase treatment, though, the cognate multimer binding on activated OT-I cells was slightly lower than that on naive cells, most likely due to the fact that TCR levels had not completely recovered to naive levels (Fig. 5).

The effect on non-cognate binding was even more dramatic. As expected, activated OT-I cells showed inefficient non-cognate multimer binding compared with naive OT-I cells, yet neuraminidase treatment disproportionately enhanced non-cognate multimer binding to the activated T cells, such that desialylated activated and naive OT-I cells now had similar staining intensities (Fig. 5).

In additional experiments, we found that this enhancing effect of desialylation on non-cognate CD8 binding also applied to cells at days 1 and 2 of activation (Fig. 6). As we have previously shown, binding of both cognate and non-cognate Class I MHC ligands can be enhanced by the anti-CD $8 \alpha$ antibody 53-6.7 $(10,23)$, which may be due to the stabilization of a Class I 'receptive' conformation of CD8 (36). When included in staining assays of activated OT-I cells, 536.7 increased non-cognate multimer binding, but still did not fully compensate for the loss of non-cognate multimer binding seen with OT-I activation (Fig. 6). Interestingly, however, 

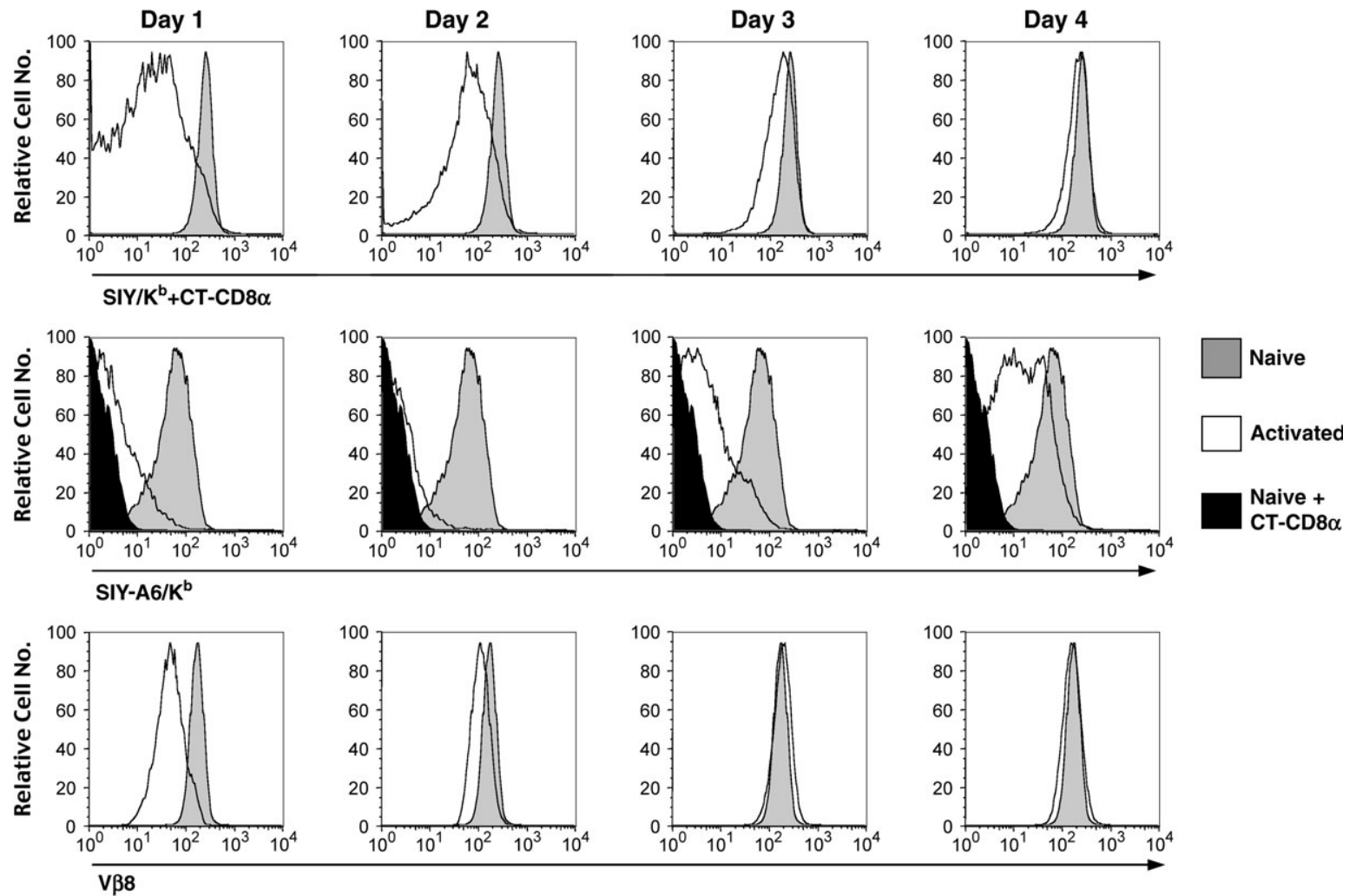

Fig. 4. Activated $2 \mathrm{C} C D 8+T$ cells show loss in CD8-dependent and -independent peptide/MHC multimer binding. Bulk lymph node $2 \mathrm{C}$ cells were activated in vitro with $1 \mu \mathrm{M}$ of SIY peptide and assessed daily from days 1 to 4 for binding to multimers and TCR levels. The top row shows binding to high-affinity SIY $/ \mathrm{K}^{\mathrm{b}}$ multimers in the presence of the blocking anti-CD8 $\alpha$ antibody, CT-CD8 $\alpha$, to assess TCR avidity alone in the absence of CD8 co-receptor contribution. The middle row shows binding to low-affinity SIY-A6/K ${ }^{b}$ multimers with or without CT-CD8 $\alpha$. The bottom panels show TCR levels using a V $\beta 8$-specific antibody. All multimers were used at a concentration of $10 \mu \mathrm{g} \mathrm{ml}^{-1}$.

a combination of neuraminidase treatment and staining with the 53-6.7 antibody restored non-cognate multimer staining on activated OT-I cells to the levels seen on naive OT-I cells (Fig. 6). The binding of the non-cognate multimers (with or without neuraminidase treatment) was completely blocked by CT-CD8 $\alpha$ (Fig. 6) which we previously showed inhibits CD8Class I multimer binding $(10,23)$, strongly suggesting that all of the binding observed involves CD8 directly.

As reported previously, staining with PNA (a lectin which binds unsialylated core-1 O-glycans) increases on activated CD8 T cells, including OT-I $(37,38)$. Naive T cells are heavily sialylated at core-1 O-glycans residues [due to the action of ST3Gal-I $(16,39)$ ], and hence staining by PNA is low on naive cells (Fig. 6) (10, 11, 37, 38). Under our stimulation conditions, at $24 \mathrm{~h}$ of activation, PNA binding is only modestly enhanced (Fig. 6B) compared with naive cells, but PNA staining increases markedly by 48-72 h (Fig. 6B) as shown previously for OT-I cells (37). Hence, despite the fact that the cells are becoming desialylated on some O-linked residues, neuraminidase still has the capacity to enhance non-cognate multimer binding at these time points.

Overall then, these results suggest that changed sialylation may account for at least some of the defective non-cognate and cognate multimer binding which accompanies $\mathrm{T}$ cell stimulation. Moreover, these data suggest that neither CD8 nor
TCR molecules on activated T cells are irreversibly compromised in their capacity to bind Class I ligands.

\section{Discussion}

$\mathrm{MHC} /$ peptide multimers have revolutionized the capacity to identify $T$ lymphocytes participating in an immune response, permitting enumeration of antigen-specific $T$ cells without requiring them to survive in tissue culture or respond to antigen in vitro. However, as we describe in this report, peptide/MHC multimer staining is not simply a reflection of TCR/CD8 surface expression, but rather is strongly influenced by the activation state of the $\mathrm{T}$ cell. These changes have a profound practical significance, in that multimer binding may be a misleading method of detecting antigen-specific T cells shortly after their stimulation in a normal immune response-indeed our data would suggest that highly CD8-dependent T cells, and/or those responding with low affinity for the peptide/MHC ligand, may be undetectable using standard multimer binding assays. Similar conclusions were reached by Braciale and colleagues in an intriguing report, published during review of this manuscript, which showed loss of cognate $\mathrm{K}^{\mathrm{d}}$ multimer binding on CD8 T cells responding to influenza in vivo, despite high expression levels of TCR and CD $\alpha \beta \beta(40)$. Interestingly, the opposite may be true of staining antigen-specific CD4 T cells since data from 

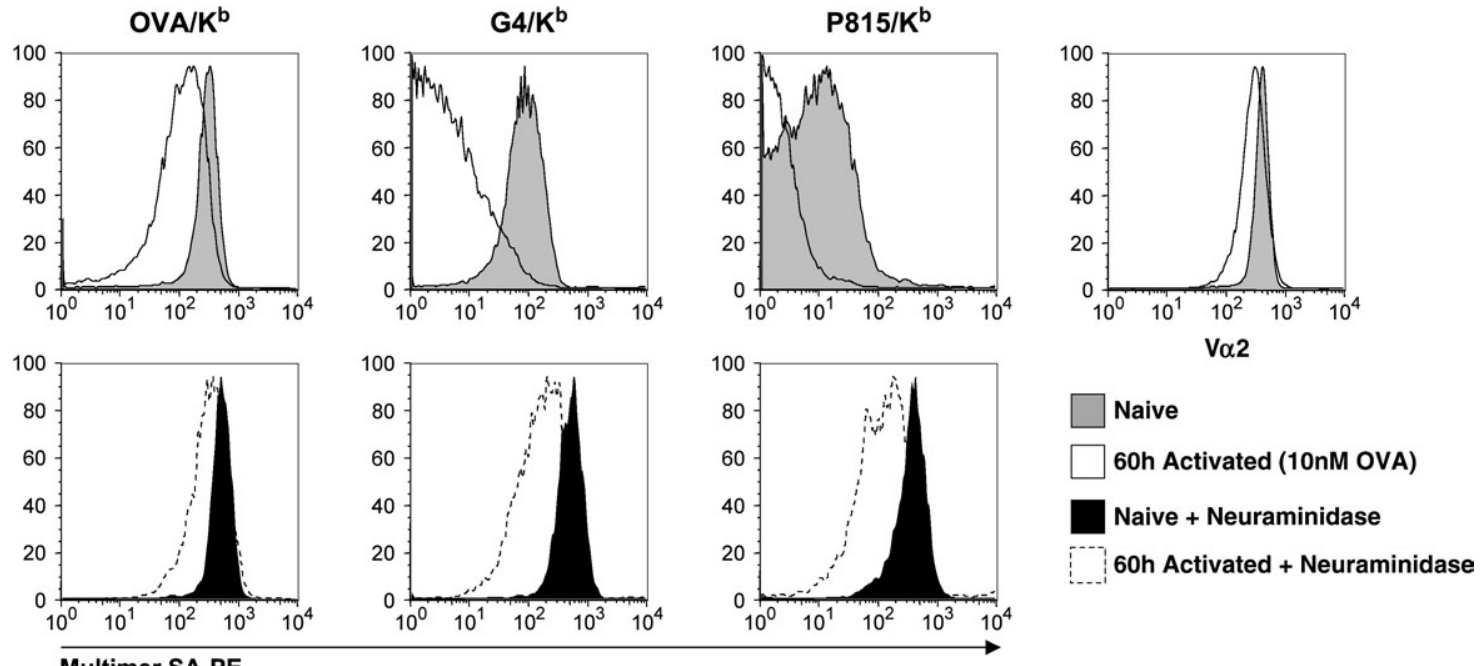

Fig. 5. Neuraminidase treatment restores both cognate and non-cognate multimer binding on activated OT-I. OT-I lymph node cells were activated in vitro for $60 \mathrm{~h}$ and stained with high-affinity cognate $\left(2 \mu \mathrm{g} \mathrm{ml} \mathrm{l}^{-1} \mathrm{OVA} / \mathrm{K}^{\mathrm{b}}\right)$, low-affinity cognate $\left(10 \mu \mathrm{g} \mathrm{ml}{ }^{-1} \mathrm{G} 4 / \mathrm{K}^{\mathrm{b}}\right)$, or non-cognate $\left(50 \mu \mathrm{g} \mathrm{ml} \mathrm{l}^{-1}\right.$ $\mathrm{P} 815 / \mathrm{K}^{\mathrm{b}}$ ) multimers. Histograms compare levels between naive and activated OT-I CD8+ Tcells. Top and lower rows show staining on cells before and after neuraminidase treatment, respectively. The extent of TCR down-regulation is also shown, by staining for $\mathrm{V} \alpha 2$ (right hand panel).

Cameron et al. (41) indicated that efficient Class II MHC multimer staining of CD4 $\mathrm{T}$ cells requires activation-induced multimer internalization.

We observe that $\mathrm{T}$ cell activation leads to dramatic and rapid loss in specific peptide/MHC multimer binding. While some loss in cognate multimer binding was predicted due to transient TCR down-regulation with activation, the loss of multimer staining does not correlate with TCR down-regulation either in its magnitude or duration. Thus we found that, for both the OT-I and 2C systems, defective cognate multimer binding was impaired on activated T cells even when TCR levels had recovered to the levels on naive cells. Furthermore, anti-TCR antibody treatment of naive T cells could block the TCR down to levels seen on activated T cells, but did not reduce cognate multimer binding to the same extent (Fig. 2). Hence, our data suggest that loss of multimer binding occurs not only in 'unusual' T cell responses [such as those directed against subdominant epitopes or during chronic T cell responses (18-22)] but this same phenomenon occurs in following conventional, acute $T$ cell activation, a conclusion reinforced by the recent report from Drake et al. (40). Our report further suggests that changes in CD8-Class I binding, in part mediated by altered cell-surface sialylation, contribute to such changes in TCR/ CD8-Class I MHC binding.

Numerous reports have shown that peptide/MHC Class I multimer binding requires coordinate recognition by both TCR and CD8 (23). Thus, it was important to determine whether loss of cognate multimer staining was due to impairment in TCR and/or CD8 engagement. Our data suggest that CD8 binding is profoundly impaired following T cell activation. TCRindependent (non-cognate) CD8 binding was virtually abolished, and CD8-dependent TCR interactions, especially with low-affinity TCR ligands, were also severely compromised. Analysis of CD8-independent multimer binding in the $2 \mathrm{C}$ system argued that TCR binding per se was less dramatically influenced, although some impairment was noted.
We had initially expected that naive T cell activation would increase CD8 non-cognate binding, based on the work performed with CTL clones which showed poor CD8-mediated adhesion to non-cognate Class I until the cells were activated through the TCR $(8,9,42)$. Our data indicate that stimulation of naive T cells leads to the opposite pattern, in which CD8-Class I binding is diminished. Interestingly, preliminary data suggest that, consistent with published reports using Class I adhesion assays $(8,9,42)$, TCR stimulation of CTL clones leads to increased levels of non-cognate multimer binding (C.K. and S.C.J., unpublished results). Such data would argue that the regulation of CD8-Class I binding on naive versus differentiated CD8 T cells is quite different, a possibility which we are currently exploring.

Loss of multimer binding did not correlate solely with receptor expression levels, suggesting a qualitative effect on CD8/TCR capacity to bind multimers. Drake et al. (40) showed that activation induced changes in cell-surface distribution of TCR molecules. This model may correlate with the effect of neuraminidase which we report here since previous reports have argued that desialylation can influence the ability of the TCR to cluster and engage peptide/MHC ligands (43). The effect of desialylation on activated T cells was unexpected, since effector $T$ cells are known to have already decreased in cell-surface sialylation (12, 15, 16), including changes in sialylation of CD8 itself following T cell activation $(13,38)$. One likely explanation for this evident discrepancy is the specificity of sialylation. There are more than a dozen murine sialyltransfersases, which differ with respect to the anomeric linkage of the sialic acid adduct as well as substrate preferences (44). Indeed, the sialyltransferase ST6GalNAc IV has been shown to be induced by activation of CD8 T cells, with similar kinetics to the effects documented here (45), and this enzyme represents a good candidate for control of the phenotype reported here. A more complex issue will be to determine whether changes in CD8 
A

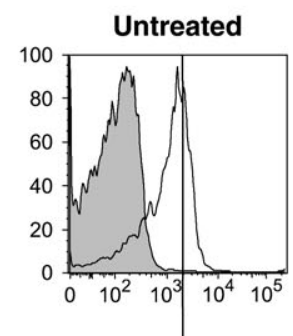

Naive

Day 1

Day 2
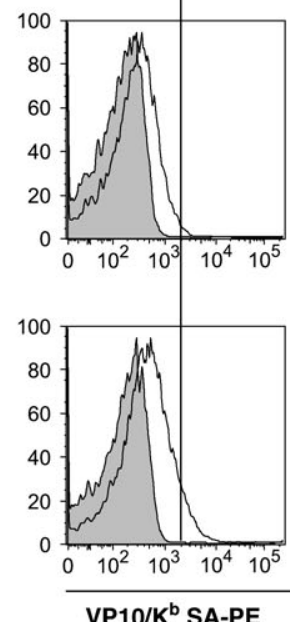

+ 53-6.7
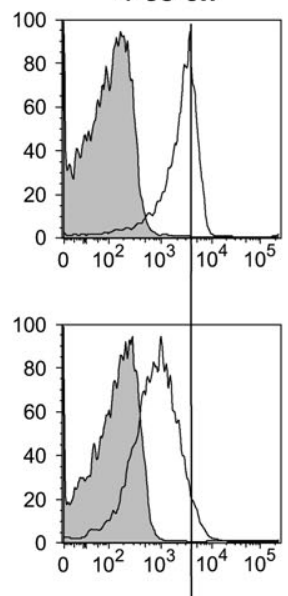
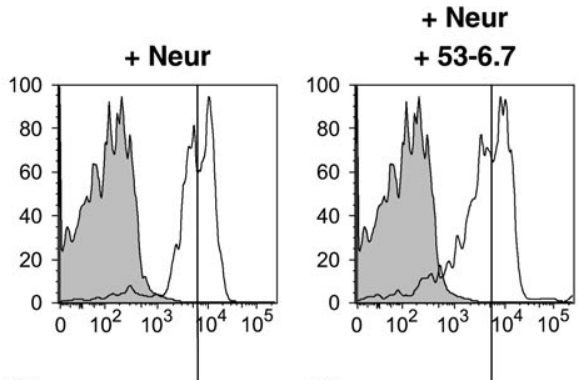

B
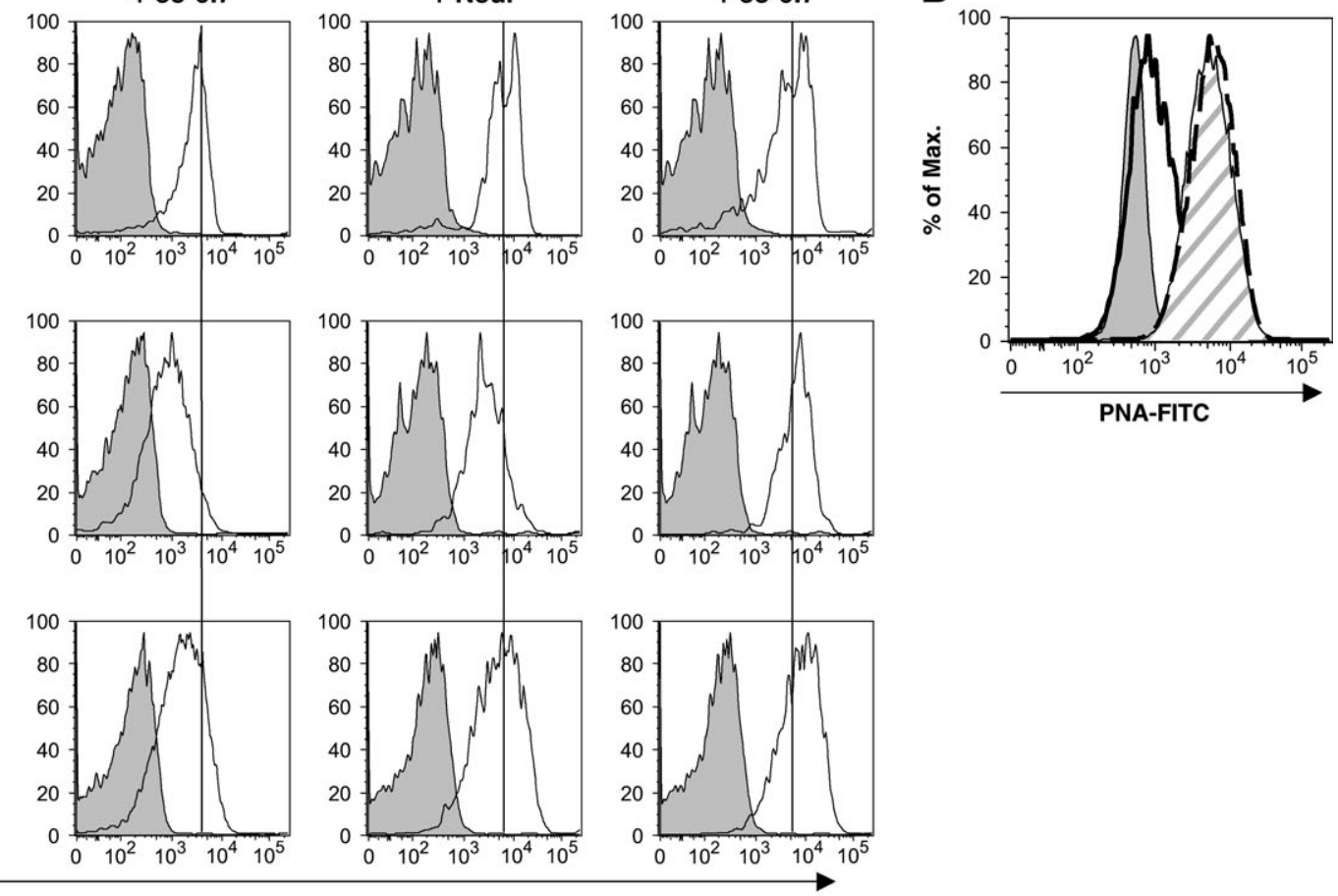

Multimer + CT-CD $8 \alpha$

Multimer

Fig. 6. Staining with the enhancing CD8 antibody 53-6.7 together with desialylation restores non-cognate CD8 binding on activated OT-I. (A) Naive and activated (days 1 and 2) OT-I cells were stained for non-cognate multimer binding similar to Fig. 5. The cells were untreated, neuraminidase treated and/or stained in the presence of the CD8 $\alpha$ antibody 53-6.7 as indicated, and multimer (VP10/K ${ }^{\mathrm{b}}$ ) staining was determined (open histogram). In addition, the untreated and neuraminidase-treated groups were stained with VP10/K $\mathrm{K}^{\mathrm{b}}$ multimer in the presence of CT-CD8 $\alpha$, which blocks CD8-Class I interactions (gray histograms), to demonstrate that neuraminidase treatment does not lead to non-specific multimer binding. Lines are shown at the mean fluorescence of the multimer staining on the naive population, to help align multimer binding on the activated cells. Multimers were used at $30 \mu \mathrm{g} \mathrm{ml}^{-1}$ (for staining with CT-CD8 $\alpha$ or without CD8 antibodies) or $10 \mu \mathrm{g} \mathrm{ml}^{-1}$ (for staining in the presence of 53-6.7). (B) PNA staining of OT-I cells after activation. Naive OT-I (shaded) and activated OT-I at days 1 (solid line), 2 (cross-hatched) or 3 (broken line) were stained with PNA-FITC.

sialylation itself plays the key role, or whether changes in other (more prominently glycosylated) $\mathrm{T}$ cell surface molecules influence the access of CD8 with its ligand, a model which has been proposed earlier (46).

Other changes might also regulate CD8 (and/or TCR) ligand binding by activated T cells. An interesting mechanism is suggested by the recent report which proposed the production of CD8 $\alpha \alpha$ homodimers on activated CD8 T cells (47). CD8 $\alpha \alpha$ binds well to the non-classical Class I molecule TL, but poorly to classical Class I MHC molecules $(11,24,48)$, hence such a change might contribute to the loss of $\mathrm{K}^{\mathrm{b}}$ multimer binding studied here. However, the report from Madakamutil et al., and our own unpublished studies using $\mathrm{TL}$ multimers (kindly provided by $\mathrm{H}$. Cheroutre) suggest that up-regulation of CD8 $\alpha \alpha$ expression is detected at $48 \mathrm{~h}$ but not at $24 \mathrm{~h}$ (data not shown), whereas we see profound impairment of cognate and non-cognate binding by day 1 of activation (Figs 3, 5 and 6). Thus, although appearance of the TL-binding form of CD8 may play a role at later stages of activation, this effect appears not to account for the early loss in classical Class I multimer binding we report here.

But what might be the physiological role for this change in Class I MHC binding? Loss of binding to specific peptide/ $\mathrm{MHC}$ ligands might seem counterintuitive, given the functional sensitivity of CTLs. Indeed, previous data from Schneck and colleagues suggested that effector CTLs were superior to naive cells in binding bivalent cognate peptide/MHC ligands at low density (7). This may relate simply to the valency of the peptide/MHC ligands used in these studies. It is interesting to note however, that Fahmy et al. used $2 \mathrm{C}$ T cells at $>\mathrm{d} 4$ postactivation, and exclusively studied binding of high-affinity cognate ligands. In our hands, such binding is largely CD8 independent and is mostly restored by that time point (Fig. 4). Thus, although we have been unable to observe enhanced multimer binding on activated T cells, such effects may be more subtle than the large-scale loss in multimer binding we observe at earlier activation time points. Furthermore, we observe a greater impact of activation on binding to lower affinity cognate ligands. For both the OT-I system $\left(\mathrm{G} 4 / \mathrm{K}^{\mathrm{b}}\right.$ binding, 
Fig. 1) and $2 \mathrm{C}\left(\mathrm{SIY}-\mathrm{A} 6 / \mathrm{K}^{\mathrm{b}}\right.$ binding, Fig. 4), activated T cells suffered a profound and sustained loss in binding to these types of ligands. Hence, these data do not necessarily disagree with the report by Fahmy et al., but suggest that enhanced peptide/MHC recognition by effector $\mathrm{T}$ cells is preceded by stage of reduced sensitivity.

The changes in peptide/MHC ligand binding which we observe would be expected to have functional consequences. Indeed, recent data from Braciale's group suggest that tetramer ${ }^{\text {low }}$ cells are altered in their ability to respond to TCR engagement, showing depressed calcium flux, IFN- $\gamma$ synthesis and degranulation (40). Our data on the decreased ability of activated $\mathrm{T}$ cells to engage low-affinity peptide/MHC ligands suggests additional consequences. The first is that activated T cells with the lowest affinity for a stimulatory foreign peptide/MHC ligand will disproportionately lose sensitivity to it. These clones would therefore fail to receive further stimulation by the antigen and may be outcompeted by more 'fit' clones (i.e. in this context, T cells bearing TCRs with higher affinity for the foreign peptide/MHC ligand). Furthermore, loss of binding to low-affinity peptide/MHC ligands may prevent recognition of self-peptide/MHC ligands. Numerous experiments suggest that naive $\mathrm{T}$ cells engage self-peptide-MHC complexes, resulting in basal TCR signaling, which may influence $T$ cell homeostasis (49-51). However, were these interactions to be maintained in the effector T cell population, there is grave potential for autoaggressive responses. Hence, activation-induced desensitization to these (low affinity) selfligands may be beneficial for the host.

Alternatively, it has long been appreciated that repeated TCR stimulation of T cells can lead to activation-induced cell death, hence the loss of peptide/MHC ligand binding we report may enhance effector survival. A distinct element in this argument is suggested by data from Potter's group, showing immature thymocyte sensitivity to apoptosis induced by non-cognate CD8 interactions (52). It is possible that loss of non-cognate binding protects highly vulnerable activated CD8 T cells from apoptosis induced in the same way, a possibility we are currently exploring. Clearly, further experiments will be needed to elucidate the consequences of decreased peptide-MHC interactions on activated CD8 $T$ cells.

In summary, our data suggest that CD8 T cells pass through a stage of depressed CD8/TCR binding following activation, which may influence the fate of the activated T cells and selection for the most robust responder cells. It is interesting to note that TCR engagement during positive selection in the thymus also leads to decreased TCR/CD8 binding and sensitivity, and that this process is also regulated by changes in sialylation. Such data suggest that $T$ cells have an unexpectedly large dynamic range in the ability of their receptors to engage peptide/ $\mathrm{MHC}$ receptors during differentiation.

\section{Acknowledgements}

We are grateful to Don Drake and Tom Braciale for communication of data ahead of publication and numerous helpful discussions, Matt Mescher and members of the Jameson/Hogquist laboratories for their valuable input and Larry Pease (Mayo Clinic, Rochester, MN, USA) for a timely gift of $2 \mathrm{C}$ mice. This work was supported by National Institutes of Health grant Al52163 (to S.C.J.) and pre-doctoral training grant awards T32 Al007313 (to both C.K. and M.A.D.).

\section{Abbreviations}

$\begin{array}{ll}\text { OVA } & \text { ovalbumin peptide } \\ \text { PNA } & \text { peanut agglutinin } \\ \text { PE } & \text { Phycoerythrin } \\ \text { SA } & \text { streptavidin }\end{array}$

\section{References}

1 Veiga-Fernandes, H., Walter, U., Bourgeois, C., McLean, A. and Rocha, B. 2000. Response of naive and memory CD8+ T cells to antigen stimulation in vivo. Nat. Immunol. 1:47.

2 lezzi, G., Karjalainen, K. and Lanzavecchia, A. 1998. The duration of antigenic stimulation determines the fate of naive and effector T cells. Immunity 8:89

3 Bachmann, M. F., Gallimore, A., Linkert, S. et al. 1999. Developmental regulation of Lck targeting to the CD8 coreceptor controls signaling in naive and memory T cells. J. Exp. Med. 189:1521.

4 Amrani, A., Serra, P., Yamanouchi, J. et al. 2001. Expansion of the antigenic repertoire of a single Tcell receptor upon Tcell activation. J. Immunol. 167:655.

5 Sykulev, Y., Joo, M., Vturina, I., Tsomides, T. J. and Eisen, H. N. 1996. Evidence that a single peptide-MHC complex on a target cell can elicit a cytolytic T cell response. Immunity 4:565.

6 Purbhoo, M. A., Irvine, D. J., Huppa, J. B. and Davis, M. M. 2004. $T$ cell killing does not require the formation of a stable mature immunological synapse. Nat. Immunol. 5:524.

7 Fahmy, T. M., Bieler, J. G., Edidin, M. and Schneck, J. P. 2001. Increased TCR avidity after T cell activation: a mechanism for sensing low-density antigen. Immunity 14:135.

8 O'Rourke, A. M., Rogers, J. and Mescher, M. F. 1990. Activated CD8 binding to class I protein mediated by the T-cell receptor results in signalling. Nature 346:187.

9 Shen, L., Potter, T. A. and Kane, K. P. 1996. Glu227-Lys substitution in the acidic loop of major histocompatibility complex class I $\alpha 3$ domain distinguishes low avidity CD8 coreceptor and avidityenhanced CD8 accessory functions. J. Exp. Med. 184:1671.

10 Daniels, M. A., Devine, L., Miller, J. D. et al. 2001. CD8 binding to $\mathrm{MHC}$ class I molecules is influenced by $\mathrm{T}$ cell maturation and glycosylation. Immunity 15:1051.

11 Moody, A. M., Chui, D., Reche, P. A., Priatel, J. J., Marth, J. D. and Reinherz, E. L. 2001. Developmentally regulated glycosylation of the CD $\alpha \alpha \beta$ coreceptor stalk modulates ligand binding. Cell 107:501.

12 Chervenak, R. and Cohen, J. J. 1982. Peanut lectin binding as a marker for activated T-lineage lymphocytes. Thymus 4:61.

13 Casabo, L. G., Mamalaki, C., Kioussis, D. and Zamoyska, R. 1994. T cell activation results in physical modification of the mouse CD8 beta chain. J. Immunol. 152:397.

14 Harrington, L. E., Galvan, M., Baum, L. G., Altman, J. D. and Ahmed, R. 2000. Differentiating between memory and effector CD8 T cells by altered expression of cell surface O-glycans. J. Exp. Med. 191:1241.

15 Galvan, M., Murali-Krishna, K., Ming, L. L., Baum, L. and Ahmed, R. 1998. Alterations in cell surface carbohydrates on Tcells from virally infected mice can distinguish effector/memory CD8+ T cells from naive cells. J. Immunol. 161:641.

16 Priatel, J. J., Chui, D., Hiraoka, N. et al. 2000. The ST3Gal-I sialyltransferase controls CD8+ T lymphocyte homeostasis by modulating O-glycan biosynthesis. Immunity 12:273.

17 Valitutti, S., Muller, S., Cella, M., Padovan, E. and Lanzavecchia, A. 1995. Serial triggering of many T-cell receptors by a few peptideMHC complexes. Nature 375:148.

18 Blohm, U., Roth, E., Brommer, K., Dumrese, T., Rosenthal, F. M. and Pircher, H. 2002. Lack of effector cell function and altered 
tetramer binding of tumor-infiltrating lymphocytes. J. Immunol. 169:5522.

19 Demotte, N., Colau, D., Ottaviani, S. et al. 2002. A reversible functional defect of CD8+ T lymphocytes involving loss of tetramer labeling. Eur. J. Immunol. 32:1688.

20 Reignat, S., Webster, G. J., Brown, D. et al. 2002. Escaping high viral load exhaustion: CD8 cells with altered tetramer binding in chronic hepatitis B virus infection. J. Exp. Med. 195:1089.

21 Spencer, J. V. and Braciale, T. J. 2000. Incomplete CD8(+) T lymphocyte differentiation as a mechanism for subdominant cytotoxic T lymphocyte responses to a viral antigen. J. Exp. Med. 191:1687.

22 Drake, D. R., III and Braciale, T. J. 2001. Cutting edge: lipid raft integrity affects the efficiency of MHC class I tetramer binding and cell surface TCR arrangement on CD8+ T cells. J. Immunol. 166:7009.

23 Daniels, M. A. and Jameson, S. C. 2000. Critical role for CD8 in T cell receptor binding and activation by peptide/major histocompatibility complex multimers. J. Exp. Med. 191:335

24 Bosselut, R., Kubo, S., Guinter, T. et al. 2000. Role of CD8beta domains in CD8 coreceptor function: importance for MHC I binding, signaling, and positive selection of CD8+ T cells in the thymus. Immunity 12:409.

25 Moody, A. M., Xiong, Y., Chang, H. C. and Reinherz, E. L. 2001. The CD8 $\alpha \beta$ co-receptor on double-positive thymocytes binds with differing affinities to the products of distinct class I MHC loci. Eur. J. Immunol. 31:2791.

26 Busch, D. H., Pilip, I. M., Vijh, S. and Pamer, E. G. 1998. Coordinate regulation of complex $T$ cell populations responding to bacterial infection. Immunity 8:353.

27 Altman, J. D., Moss, P. A. H., Goulder, P. J. R. et al. 1996. Phenotypic analysis of antigen-specific T lymphocytes. Science 274:94

28 Whelan, J. A., Dunbar, P. R., Price, D. A. et al. 1999. Specificity of CTL interactions with peptide-MHC class I tetrameric complexes is temperature dependent. J. Immunol. 163:4342.

29 Salio, M., Valitutti, S. and Lanzavecchia, A. 1997. Agonist-induced $T$ cell receptor down-regulation: molecular requirements and dissociation from T cell activation. Eur. J. Immunol. 27:1769.

30 Liu, H., Rhodes, M., Wiest, D. L. and Vignali, D. A. 2000. On the dynamics of TCR:CD3 complex cell surface expression and downmodulation. Immunity 13:665.

31 Rosette, C., Werlen, G., Daniels, M. A. et al. 2001. The impact of duration versus extent of TCR occupancy on T cell activation: a revision of the kinetic proofreading model. Immunity 15:59.

32 Block, M. S., Johnson, A. J., Mendez-Fernandez, Y. and Pease, L. R. 2001. Monomeric class I molecules mediate TCR/CD3\&/ CD8 interaction on the surface of T cells. J. Immunol. 167:821.

33 Daniels, M. A., Schober, S. L., Hogquist, K. A. and Jameson, S. C 1999. Cutting edge: a test of the dominant negative signal model for TCR antagonism. J. Immunol. 162:3761.

34 Holman, P. O., Walsh, E. R. and Jameson, S. C. 2005 Characterizing the impact of CD8 antibodies on class I MHC multimer binding. J. Immunol. 174:3986.

35 Wu, W., Harley, P. H., Punt, J. A., Sharrow, S. O. and Kearse, K. P. 1996. Identification of CD8 as a peanut agglutinin (PNA) receptor molecule on immature thymocytes. J. Exp. Med. 184:759.
36 Devine, L., Hodsdon, M. E., Daniels, M. A., Jameson, S. C. and Kavathas, P. B. 2004. Location of the epitope for an anti-CD8alpha antibody 53.6.7 which enhances CD8alpha-MHC class I interaction indicates antibody stabilization of a higher affinity CD8 conformation. Immunol. Lett. 93:123.

37 Pappu, B. P. and Shrikant, P. A. 2004. Alteration of cell surface sialylation regulates antigen-induced naive CD8+ Tcell responses. J. Immunol. 173:275.

38 Amado, M., Yan, Q., Comelli, E. M., Collins, B. E. and Paulson, J. C. 2004. Peanut agglutinin high phenotype of activated CD8+ T cells results from de novo synthesis of CD45 glycans. J. Biol. Chem. 279:36689.

39 Martin, L. T., Marth, J. D., Varki, A. and Varki, N. M. 2002. Genetically altered mice with different sialyltransferase deficiencies show tissue-specific alterations in sialylation and sialic acid 9-O-acetylation. J. Biol. Chem. 277:32930.

40 Drake, D. R., III, Ream, R. M., Lawrence, C. W. and Braciale, T. J. 2005. Transient loss of MHC class I tetramer binding after CD8+ T cell activation reflects altered T cell effector function. J. Immunol. 175:1507.

41 Cameron, T. O., Cochran, J. R., Yassine-Diab, B., Sekaly, R. P. and Stern, L. J. 2001. Cutting edge: detection of antigen-specific CD4+ T cells by HLA-DR1 oligomers is dependent on the T cell activation state. J. Immunol. 166:741.

42 Kane, K. P. and Mescher, M. F. 1993. Activation of CD8-dependent cytotoxic $T$ lymphocyte adhesion and degranulation by peptide class I antigen complexes. J. Immunol. 150:4788.

43 Mekala, D. J. and Geiger, T. L. 2003. Functional segregation of the TCR and antigen-MHC complexes on the surface of CTL. J. Immunol. 171:4089.

44 Varki, A., Cummings, R., Esko, J., Freeze, H., Hart, G. and Marth, J. 1999. Essentials of Glycobiology. Cold Spring Harbor Laboratory Press, Cold Spring Harbor, New York.

45 Kaufmann, M., Blaser, C., Takashima, S., Schwartz-Albiez, R., Tsuji, S. and Pircher, H. 1999. Identification of an alpha2,6sialyltransferase induced early after lymphocyte activation. Int. Immunol. 11:731.

46 Baum, L. G. 2002. Developing a taste for sweets. Immunity 16:5.

47 Madakamutil, L. T., Christen, U., Lena, C. J. et al. 2004. CD8 $\alpha \alpha-$ mediated survival and differentiation of CD8 memory $\mathrm{T}$ cell precursors. Science 304:590.

48 Leishman, A. J., Naidenko, O. V., Attinger, A. et al. 2001. T cell responses modulated through interaction between CD8 $\alpha \alpha$ and the nonclassical MHC class I molecule, TL. Science 294:1936.

49 Ge, Q., Bai, A., Shen, C. H., Eisen, H. N. and Chen, J. 2003. CD4+ T-cell responses to self-peptide-MHC. Trends Immunol. 24:186.

50 Goldrath, A. W. and Bevan, M. J. 1999. Selecting and maintaining a diverse T-cell repertoire. Nature 402:255.

51 Hogquist, K. A., Starr, T. K. and Jameson, S. C. 2003. Receptor sensitivity: when T cells lose their sense of self. Curr. Biol. 13:R239.

52 Grebe, K. M., Clarke, R. L. and Potter, T. A. 2004. Ligation of CD8 leads to apoptosis of thymocytes that have not undergone positive selection. Proc. Natl Acad. Sci. USA 101:10410. 